\title{
Nutrición pre y posnatal: impacto a largo plazo en la salud
}

\author{
Prenatal and postnatal nutrition: long term impact on health
}

\author{
Dra. María del Carmen Toca ${ }^{a}$, Dra. Miriam Tonietti ${ }^{b}$ y Dra. Carmen Vecchiarellic
}

\begin{abstract}
RESUMEN
Lanutrición en etapas tempranas dela vida es uno de los factores ambientales de mayor influencia para el desarrollo y futura salud de órganos y sistemas. Mecanismos epigenéticos han podido explicar cómo la exposición nutricional pre- $y$ posnatal puede afectar la expresión genética con el subsecuente riesgo de enfermedades inmunes y metabólicas.

El objetivo del presente trabajo es actualizar el conocimiento del impacto del estado nutricional y patrones dietarios de la madre durante la gestación y de la alimentación del niño durante el primer año sobre el riesgo de desarrollo de enfermedades futuras.

Palabras clave: nutrición, embarazo, lactancia, alimentación complementaria, microbiota.
\end{abstract}

\section{ABSTRACT}

Nutrition in early stages of life is one of the most influential environmental factors for the good development of organs and systems and the wellbeing of the child. Epigenetic mechanisms can explain how prenatal and postnatal nutrition affects genes expression with the subsequent risk of immune and metabolic diseases.

The objective of this paper is to update the knowledge of the role the nutritional status and dietary practices of pregnant women and the child's feeding patterns over the first year of life have in the risk of future diseases.

Key words: nutrition, pregnancy, lactation, complementary feeding, microbiota.

http:/ /dx.doi.org/10.5546/aap.2015.248

\section{INTRODUCCIÓN}

La nutrición prenatal y posnatal son los factores ambientales de mayor influencia durante el desarrollo fetal e infantil, fundamentales para el desarrollo madurativo de órganos y su óptima función fisiológica.

Las exposiciones ambientales durante estos períodos sensibles de la vida pueden determinar cambios permanentes en muchos procesos fisiológicos, conocidos como "programming".

Es reconocido el efecto de factores nutricionales y otras exposiciones ambientales sobre la expresión génica, que determinan variantes fenotípicas. Los mecanismos epigenéticos pueden ser definidos como una red de procesos biológicos que regulan la expresión de genes y que producen cambios en la función celular sin cambiar la secuencia del ADN. La modulación de la expresión genética a través de cambios epigenéticos es un importante mecanismo por el cual la exposición dietaria puede generar cambios en el desarrollo inmune, en la expresión inmunogenética, lo cual puede, potencialmente, producir efectos sobre la función inmune, el fenotipo clínico y el riesgo a desarrollar enfermedades. ${ }^{1}$

En los últimos años, se demostró el impacto de los patrones nutricionales en la vida temprana sobre el riesgo cardiovascular y el desarrollo de enfermedades metabólicas en la vida adulta. $^{1}$

Los cambios ambientales y del estilo de vida se han incorporado como factores que inciden en el aumento de las enfermedades crónicas no trasmisibles (ECNT): enfermedad cardiovascular, obesidad y diabetes tipo 2, algunos cánceres, enfermedad pulmonar crónica, enfermedades inmunes y alérgicas. De todos los potenciales factores ambientales implicados, los cambios modernos en la alimentación son los que confieren mayor riesgo tanto para el desarrollo de enfermedades alérgicas como metabólicas. ${ }^{2,3}$ Aunque hay amplias variaciones regionales, muchas dietas modernas contienen alimentos más procesados y sintéticos, ricos en grasas y carbohidratos refinados, con pocas cantidades de fibra, pescado fresco, frutas y vegetales en comparación con las dietas tradicionales. Estos cambios alimentarios han sido asociados 
a cambios en la microbiota intestinal, en la respuesta metabólica y la función inmune porque condicionan un estado de inflamación crónica de bajo grado, con alteración de mecanismos homeostáticos y, por lo tanto, como factores de mayor riesgo para las ECNT. ${ }^{3-6}$

El objetivo del presente trabajo es revisar y actualizar el conocimiento del impacto del estado nutricional y de los patrones dietarios de la madre durante la gestación y de la alimentación del niño en el primer año de vida sobre el riesgo de desarrollo de enfermedades futuras.

\section{Alimentación durante el embarazo y su influencia en la modulación inmune posnatal Rol epigenético}

Aunque se reconoce la importancia de los factores genéticos en la predisposición a enfermedades, el drástico aumento de las ECNT no puede ser explicado solamente por esta predisposición genética. La etapa prenatal es un período crítico en la determinación de la posterior ontogénesis inmune.

Estudios epidemiológicos muestran la importancia de patrones dietarios favorables, tales como la "dieta mediterránea", durante el embarazo y la niñez temprana, en función de la protección del desarrollo de enfermedades alergénicas. También han sido asociados con la reducción del riesgo de ECNT en etapas posteriores de la vida. Estos estudios han demostrado las propiedades inmunomoduladoras específicas entre algunos componentes de la dieta, como vitaminas, minerales y ácidos grasos polinsaturados de cadena larga (AGCL) $\omega 3 \mathrm{y}$ $\omega 6 .^{1,7-9}$

Los AGCL son un buen ejemplo de factores dietarios que tienen múltiples funciones metabólicas y estructurales que pueden modular la respuesta inflamatoria. ${ }^{1}$

En humanos, altas dosis de suplementos con $\omega-3$ AGCL en el embarazo pueden modular el estrés oxidativo fetal y el metabolismo de los leucotrienos, con efectos asociados sobre la función inmune. ${ }^{1}$

Estudios clínicos de suplementación con aceite de pescado durante el embarazo y el período posnatal temprano han sido asociados con una reducción de enfermedades alérgicas y con importante efecto benéfico biológico en el desarrollo cognitivo y visual. ${ }^{1,10}$

Se recomienda una ingesta habitual de aceite de pescado, pescados de mar o sus suplementos, incluso desde antes de la concepción, ya que asegura la acumulación de depósitos de $\omega$-3 AGCL, que serán útiles posteriormente durante el embarazo y la lactancia.

Otros nutrientes de la dieta con efecto inmunomodulador son los antioxidantes (selenio, zinc, vitamina $C$ y vitamina $E$ ). Su ingesta alta durante el embarazo y primeros meses de la vida reducen el riesgo de eczemas, sibilancias y asma. ${ }^{1}$

Recientes estudios han demostrado la importancia del desarrollo de la microbiota intestinal (MI) en el período posnatal inmediato. La constitución de la MI está determinada por la MI materna, el tipo de nacimiento y el tipo de alimentación inicial (leche humana o fórmulas). Las modificaciones posteriores dependerán del medio ambiente (internación neonatal o su hogar), el uso de antibióticos, antiácidos y las condiciones de higiene. A posteriori, dietas con alto contenido graso y bajas en fibra, poco saludables, alteran la MI. La leche materna, el uso de prebióticos y dietas ricas en pescados de mar, fibras, vegetales y frutas, en cambio, promueven una MI saludable.

La MI interactúa con el sistema inmune e influencia el desarrollo de marcas epigenéticas en el huésped. Los ácidos grasos de cadena corta, producto de la fermentación de la MI sobre la fibra de la dieta, así como también la vitamina D y los folatos, son ejemplos de nutrientes que modifican la expresión de genes y la respuesta inmune. ${ }^{11-19}$

\section{Estado nutricional de la mujer embarazada. Impacto en la salud del hijo}

El cuidado antenatal es una oportunidad única de llegar a un gran número de mujeres sanas, con la posibilidad de influir en la salud de la próxima generación.

El hábito de patrones dietarios saludables desde antes del embarazo demostró estar asociado con una reducción de entre el 24 y el $46 \%$ de la diabetes gestacional. ${ }^{20}$

La ganancia inadecuada de peso durante el embarazo se asocia a un riesgo aumentado para la salud de la madre y del hijo.

La obesidad en mujeres embarazadas se asocia a cambios inflamatorios y metabólicos, que impactan sobre el desarrollo fetal. . $^{21,22}$

El déficit de macro y/o micronutrientes durante el embarazo, y el tiempo de duración de este, puede determinar un incremento en la morbimortalidad materno-fetal, que se expresa como recién nacidos con bajo peso al nacer, prematuros y/o restricción de crecimiento intrauterino. ${ }^{7}$ Para sobrevivir 
a tales noxas, el feto responde con cambios programados a nivel cardiológico, metabólico, endócrino y epigenético (adaptación fetal), que pueden predisponer luego, a lo largo de la vida, al desarrollo de hipertensión, enfermedad coronaria, resistencia a la insulina, diabetes, hiperlipidemia y obesidad. ${ }^{9}$

\section{La nutrición del lactante y su efecto sobre la salud futura \\ Lactancia materna}

La leche materna es considerada un alimento funcional. Estos son alimentos modificados o ingredientes alimentarios que proveen beneficio a la salud a través de alguno o algunos de sus componentes. ${ }^{23}$

Según la composición y el momento, denominamos calostro a la leche humana (LH) que surge desde el $5^{\text {to }}$ mes de embarazo; leche de transición, a la que se produce entre los 4 y los 15 días del nacimiento; y leche madura o definitiva, luego de las 2 semanas del nacimiento.

Las características de la LH, en especial la composición del calostro, son de excelencia. En el calostro obtenido de una madre que tuvo un nacimiento prematuro, los nutrientes presentan diferentes concentraciones comparadas con las del calostro de una madre con un nacimiento a término y se adecuan a las necesidades del niño prematuro (crecimiento e inmunidad).

La LH juega un rol fundamental sobre el desarrollo del sistema inmune a través de su composición porque aporta inmunoglobulinas, lactoferrina, lisozimas, citocinas IL4, linfocitos $\mathrm{T}$ helper2, TGF $\beta$ y linfocitos $\mathrm{T}$ regulatorios, y porque contiene un alto porcentaje de oligosacáridos ( $\beta$ GOS) con función prebiótica y bacterias probióticas (Bifidobacterias $10^{3}$ colonias), que favorecen el desarrollo de una MI beneficiosa. ${ }^{24,25}$

Contribuye al vínculo madre-hijo, es económica y no requiere preparación.

Contiene AGCL $\omega-3$ docosahexanoico (DHA) y w-6 ácido araquidónico (ARA), que actúan directamente o como precursores de moléculas que modulan el crecimiento celular, la comunicación inter e intracelular y la función proteica. Son constituyentes de la membrana fosfolipídica y regulan su función. Predominan en el tejido nervioso; el DHA se acumula selectivamente en la retina y la sustancia gris; favorecen el desarrollo neurológico, intelectual y visual. La depleción reduce la función visual; genera alteraciones cognitivas y conductuales, como también alteraciones del metabolismo de los neurotransmisores, disminución de la actividad de membranas y de receptores. ${ }^{10,25}$

La LH también brinda una alta biodisponibilidad del hierro y una relación $\mathrm{Ca} / \mathrm{P}$ (calcio 2: fósforo 1) que favorece la absorción. ${ }^{26}$

La lactancia previene múltiples enfermedades y situaciones clínicas: enfermedades infecciosas virales o bacterianas; atenúa las infecciones en curso, diarrea, anemia, obesidad, diabetes, enfermedades cardiovasculares, enfermedades oncológicas, muerte súbita y cáncer de mama.

La Organización Mundial de la Salud (OMS) promueve la lactancia exclusiva durante los primeros seis meses de vida y recomienda prolongarla el mayor tiempo posible, hasta los 2 años o más.

Cuando la lactancia no es posible, o cuando es indispensable complementarla, es necesario recurrir a diferentes fórmulas, que deben ser adecuadas para cada edad del niño. Las fórmulas de inicio se indican en lactantes sanos, desde el nacimiento hasta los 6 meses. Las fórmulas de continuación, a partir de los 6 meses y hasta el año o más, coincidentemente con la alimentación con semisólidos y sólidos.

Estas fórmulas se presentan fluidas o en polvo. Las leches en polvo no son estériles, ya que, en los procesos de fabricación, si bien se realizan múltiples controles para disminuir la presencia de microorganismos, no es posible lograr la ausencia total de gérmenes.

En grupos de riesgo, como son los recién nacidos prematuros, recién nacidos de bajo peso al nacer, desnutridos e inmunocomprometidos, es recomendable la presentación líquida. ${ }^{27,28}$

La recomendación de la Academia Americana de Pediatría es postergar el consumo de leche de vaca hasta después del primer año de edad, ya que la ingesta de leche de vaca sin modificar durante el primer año se asocia con anemia, elevada carga renal de solutos e inadecuado aporte y proporción de $\mathrm{Ca} / \mathrm{P}$.

Varios son los beneficios reconocidos en el uso de fórmulas lácteas versus leche de vaca sin modificar, como la adecuada proporción de AGCL, la menor carga proteica (similar a la LH), el aporte de vitaminas, minerales y micronutrientes, su contenido de prebióticos y probióticos, que favorecen el desarrollo de una MI más saludable.

Se recomiendan, para los niños menores de 6 meses, fórmulas que contengan DHA y ARA para cubrir sus requerimientos diarios, con el objetivo de prevenir enfermedades alergénicas y beneficiar 
el desarrollo motor, visual y cognitivo. ${ }^{10}$

En los últimos años, además, ha tomado real importancia el control del aporte proteico. Ya desde el año 1995, se ha propuesto la hipótesis de que el alto aporte proteico en la dieta del lactante estimula un crecimiento acelerado e incrementa el riesgo de sobrepeso y obesidad. ${ }^{29}$ Esta asociación parece depender del efecto estimulador de una alta ingesta proteica sobre el factor insulino-símil IGF I, que resulta en una mayor velocidad de crecimiento y un aumento de masa muscular y masa grasa. La rápida ganancia de peso durante el primer año se asocia con mayor riesgo de obesidad a futuro en la vida. Los lactantes alimentados al pecho tienen una velocidad de crecimiento menor que los alimentados con fórmula. Es importante definir el mejor contenido proteico de la fórmula. Un estudio aleatorizado controlado doble ciego, multicéntrico, realizado en 5 países de Europa por Koletzko et al..$^{30}$ comparó el crecimiento de niños de 2 meses de edad asignados a recibir fórmula con alto $(2,9 \mathrm{~g} / 100 \mathrm{kcal})$ o bajo $(1 \mathrm{~g} / 100 \mathrm{kcal})$ contenido proteico y fórmula de continuación con $4,4 \mathrm{~g}$ prot/100 kcal vs. $2,2 \mathrm{~g}$ prot $/ 100 \mathrm{kcal}$. Los lactantes que recibieron fórmula con alta carga proteica tuvieron una mayor ganancia de peso en los primeros 2 años sin efecto sobre la talla. A los 24 meses, el puntaje $z$ de $\mathrm{P} / \mathrm{T}$ en el grupo con menor carga proteica resultó significativamente menor que en el grupo con alta carga (similar al crecimiento de los niños alimentados al pecho). Los autores concluyeron que limitar la ingesta de proteínas en la infancia podía ser un modo de disminuir el riesgo de sobrepeso y obesidad posterior. ${ }^{30,31}$

\section{Alimentación complementaria}

A partir del $6^{\text {to }}$ mes, se iniciará la alimentación complementaria (AC), por lo que se constituirá una dieta mixta: lactancia y semisólidos o fórmula y semisólidos. ${ }^{32}$

La introducción temprana de sólidos, la administración de leche de vaca antes del primer año de edad y el consumo abundante de jugos durante los primeros 2 años -todas estas, prácticas dietéticas no deseables- tienen alta prevalencia en nuestro medio.

La obesidad durante los dos primeros años de vida es considerada como un factor de predicción para la obesidad en el adulto, y datos recientes indican que las tendencias ponderales comienzan a configurarse en una fase temprana de la vida del niño. ${ }^{31}$
Se debe estimular el hábito de consumo de frutas y verduras, alimentos ricos en fibra, bajos en proteínas, carentes de grasas saturadas y relativamente de bajo valor calórico para mejorar la calidad de la alimentación. ${ }^{32}$

No se ha demostrado que la introducción tardía o la no incorporación de alimentos potencialmente alergénicos (huevos, pescado) reduzca el desarrollo de alergias, aun en los niños considerados de riesgo. Es prudente evitar la introducción temprana (en menores de 4 meses) y tardía (después de los 7 meses) del gluten; es conveniente su incorporación gradual mientras el lactante reciba alimentación al pecho, con el fin de reducir el riesgo de enfermedad celíaca, diabetes tipo 1 y alergia al trigo. ${ }^{33}$

Se recomienda utilizar hidratos de carbono complejos (tubérculos, cereales y legumbres) y evitar los azúcares simples (dulces) para no inducir a hábitos inadecuados, producir caries o aumentar el riesgo de desarrollo de sobrepeso. ${ }^{34}$

Durante los dos primeros años de vida en niños sanos, no se recomienda limitar la cantidad o el tipo de grasa de la alimentación con alimentos descremados. El aporte de fibra no debe ser elevado para evitar que interfiera en la absorción de hierro, zinc y calcio. La AC debe contener cantidades suficientes de hierro biodisponible, ya que debe cubrir más del $90 \%$ de los requerimientos de hierro. Se recomienda la incorporación de carnes de vaca o pollo desde el $6^{\text {to }}$ mes de vida y de huevo y pescado, desde el $7^{\mathrm{mo}}$ mes de edad. ${ }^{34}$

Durante esta etapa, la alimentación complementaria debe asegurar los aportes de AGCL $\omega-3$ y $\omega-6$ en proporciones equilibradas. ${ }^{10}$

En aquellos niños que, por decisión y cultura familiar, reciban una dieta vegetariana, se recomienda vigilar el aporte de suficiente cantidad de hierro y de leche $(500 \mathrm{ml}$ de $\mathrm{LH}$ o fórmula) y productos lácteos derivados. No se deben indicar dietas veganas en lactantes y niños pequeños. Esta etapa debe caracterizarse por alimentos con adecuada calidad, variedad, textura. Todos los alimentos deben estar muy bien cocidos, y las frutas, peladas y lavadas. No se debe dar miel, ni sal, ni azúcar y se deben incorporar carnes precozmente siempre muy bien cocidas. $^{34}$

\section{CONCLUSIONES}

La nutrición materna es el factor ambiental de mayor influencia sobre el crecimiento fetal y el desarrollo de todos los sistemas. De modo 
similar, la nutrición posnatal, los dos primeros años de vida, es crítica para la maduración de órganos y para su óptima función fisiológica. Cambios ambientales y en el estilo de vida han sido relacionados con el aumento de las ECNT: enfermedades cardiovasculares, obesidad y diabetes tipo 2, algunos cánceres, enfermedad pulmonar crónica, enfermedades inmunes y alérgicas. Los factores ambientales implicados, en especial los cambios modernos de dietas, son los de mayor riesgo. Estos cambios han sido asociados a modificaciones en la microbiota intestinal, la respuesta metabólica y la función inmune. La leche humana posee inigualables propiedades inmunológicas y nutricionales; favorece el desarrollo neurológico, intelectual, visual, de una microbiota saludable; y contribuye a lograr un vínculo madre-hijo ideal. La introducción temprana de sólidos (antes de los 6 meses de edad), de leche de vaca (antes del primer año de edad), el consumo abundante de jugos durante los primeros 2 años, de alimentos con alta densidad calórica, ricos en grasas saturadas y pobres en fibras son prácticas dietéticas no deseables y muy frecuentes en nuestro medio que los pediatras debemos modificar para mejorar el estado nutricional de los niños y prevenir el desarrollo posterior de enfermedades en la adultez asociadas a ellas.

\section{REFERENCIAS}

1. Amarasekera M, Prescott SL, Palmer DJ. Nutrition in early life, immune-programming and allergies: the role of epigenetics. Asian Pac J Allergy Immunol 2013;31(3):175-82.

2. Dattilo AM, Birch L, Krebs NF, Lake A, et al. Need for early interventions in the prevention of pediatric overweight: a review and upcoming directions. J Obes 2012;2012:123023.

3. Crowe KM, Francis C. Position of the Academy of Nutrition and Dietetics: functional foods. J Acad Nutr Diet 2013;113(8):1096-103.

4. Nicholson JK, Holmes E, Kinross J, Burcelin R, et al. Host-gut microbiota metabolic interactions. Science 2012;336(6086):1262-7.

5. Clemente JC, Ursell LK, Parfrey LW, Knight R. The impact of the gut microbiota on human health: an integrative view. Cell 2012;148(6):1258-70.

6. Claus SP, Ellero SL, Berger B, Krause L. Colonizationinduced host-gut microbial metabolic interaction. MBio 2011;2(2):e00271-10.

7. Anderson AS. Symposium on 'Nutritional adaptation to pregnancy and lactation'. Pregnancy as a time for dietary change? Proc Nutr Soc 2001;60(4):497-504.

8. Matusiak K, Barrett HL, Callaway LK, Nitert MD. Periconception weight loss: common sense for mothers, but what about for babies? J Obes 2014;2014:204295.

9. National Research Council. Influence of pregnancy weight on maternal and child health: workshop report. Washington DC: The National Academies Press; 2007. [Acceso: 22 de diciembre de 2014]. Disponibleen: http:/ / www.nap.edu/ catalog/11817.html

10. Koletzko B, Boey CC, Campoy C, Carlson SE, et al.
Current information and Asian perspectives on long-chain polyunsaturated fatty acids in pregnancy, lactation, and infancy: systematic review and practice recommendations from an early nutrition academy workshop. Ann Nutr Metab 2014;65(1):49-80.

11. Jiménez E, Marín ML, Martín R, Odriozola JM, et al. Is meconium from healthy newborns actually sterile? Res Microbiol 2008;159(3):187-93.

12. Mackie RI, Sghir A, Gaskins HR. Developmental microbial ecology of the neonatal gastrointestinal tract. Am J Clin Nutr 1999;69(5):1035S-45S.

13. Mändar R, Mikelsaar M. Transmission of mother's microflora to the newborn at birth. Biol Neonate 1996;69(1):30-5.

14. Domínguez-BelloMG, CostelloEK,Contreras M,Magris M, et al. Delivery mode shapes the acquisition and structure of the initial microbiota across multiple body habitats in newborns. Proc Natl Acad Sci USA 2010;107(26):11971-5.

15. Penders J, Thijs C, Vink C, Stelma FF, et al. Factors influencing the composition of the intestinal microbiota in early infancy. Pediatrics 2006;118(2):511-21.

16. Palmer C, Bik EM, DiGiulio DB, Relman DA, et al. Development of the human infant intestinal microbiota. PLoS Biol 2007;5(7):e177.

17. Saavedra JM. Use of probiotics in pediatrics: rationale, mechanisms of action, and practical aspects. Nutr Clin Pract 2007;22(3):351-65.

18. Sela DA, Chapman J, Adeuya A, Kim JH, et al. The genome sequence of Bifidobacterium longum subsp. infantis reveals adaptations for milk utilization within the infant microbiome. Proc Natl Acad Sci USA 2008;105(48):18964-9.

19. Hooper LV, Littman DR, Macpherson AJ. Interactions between the microbiota and the immune system. Science 2012;336(6086):1268-73.

20. Tanentsapf I, Heitmann BL, Adegboye AR. Systematic review of clinical trials on dietary interventions to prevent excessive weight gain during pregnancy among normal weight, overweight and obese women. BMC Pregnancy Childbirth 2011;11:81.

21. Shapira N. Prenatal nutrition: a critical window of opportunity for mother and child. Womens Health (Lond Engl) 2008;4(6):639-56.

22. Vesco KK, Dietz PM, Rizzo J, Stevens VJ, et al. Excessive gestational weight gain and postpartum weight retention among obese women. Obstet Gynecol 2009;114(5):1069-75.

23. Ferrer Lorente B, Dalmau Serra J. Alimentos funcionales: probióticos. Acta Pediatr Esp 2001;59:150-5.

24. Soto A, Martín V, Jiménez E, Mader I, et al. Lactobacilli and bifidobacteria in human breast milk: influence of antibiotherapy and other host and clinical factors. J Pediatr Gastroenterol Nutr 2014;59(1):78-88.

25. Chirdo FG, Menéndez AM, Pita Martín de Portela ML, Sosa P, et al. Prebióticos en la salud infantil. Arch Argent Pediatr 2011;109(1):49-55.

26. Section of Breastfeeding. Breastfeeding and the use of human milk. Pediatrics 2012;129(3):e827-41.

27. Vecchiarelli C. Nutrición enteral en prematuros. Revista Enfermería Neonatal 2012;IV(13):12-8.

28. Moreno Villares JM, Galiano Segovia MJ, Dalmau Serra J. Preparación y manejo de las fórmulas infantiles en polvo. Reflexiones en torno a las recomendaciones del Comité de Nutrición de la ESPGHAN. Acta Pediatr Esp 2005;63:27982.

29. Rolland-Cachera MF, Deheeger M, Akrout M, Bellisle F. Influence of macronutrients on adiposity development: a follow up study of nutrition and growth from 10 months to 8 years of age. Int J Obes Relat Metab Disord 1995;19(8):573-8.

30. Koletzko B, von Kries R, Closa R, Escribano J, et al. Lower 
protein in infant formula is associated with lower weight up to age $2 \mathrm{y}$; a randomized clinical trial. Am J Clin Nutr 2009;89(6):1836-45.

31. Koletzko B, Von Kries R, Closa R, Escribano J, et al. Can infant feeding choices modulate later obesity risk? Am J Clin Nutr 2009;89(5):1502S-8S.

32. Agostoni C, Decsi T, Fewtrell M, Goulet O, et al. Complementary feeding: a commentary by the ESPGHAN Committee on Nutrition. I Pediatr Gastroenterol Nutr
2008;46(1):99-110.

33. Cattaneo A, Williams C, Pallás-Alonso CR, HernándezAguilar MT, et al. ESPGHAN's 2008 recommendation for early introduction of complementary foods: how good is the evidence? Matern Child Nutr 2011;7(4):335-43.

34. Comité de Nutrición. Guía de alimentación para niños sanos de 0 a 2 años. Buenos Aires: Sociedad Argentina de Pediatría; 2001.

\section{Conintelagen (0)}

Los siguientes resúmenes y comentarios de trabajos seleccionados se encuentran disponibles en la versión electrónica de este número.

PEDIATRICS 2014 Dec;134(6):e1537-44

Validación de una regla de predicción clínica para el traumatismo craneano pediátrico abusivo

Validation of a clinical prediction rule for pediatric abusive head trauma

Hymel KP, et al.

Comentario: Dr. Javier Indart de Arza. Unidad de Violencia Familiar. Hospital General de Niños "Dr. Pedro de Elizalde".

ARCH DIS CHILD published online January 6, 2015

Evolución coronaria aguda y tardía en 1073 pacientes con enfermedad de Kawasaki con y sin tratamiento con $\gamma$-inmunoglobulina endovenosa

Acute and late coronary outcomes in 1073 patients with Kawasaki disease with and without intravenous $\gamma$-inmunoglobulin therapy

Ming-Tai Lin, et al.

Comentario: Dr. Luis Eduardo Urrutia.

Coordinación de Emergencia, Hospital Nacional de Pediatría Prof. Dr. Juan P. Garrahan.

PEDIATRICS 2015 Jan;135(1):e7-e15. doi: 10.1542/peds.2014-1115

El uso de Historia Clínica Electrónica por pediatras que trabajan en consultorio

Use of electronic health record systems by office-based pediatricians

Lehmann CU, et al.

Comentario: Dra. Paula Otero. Hospital Italiano de Buenos Aires.

N ENGL J MED 2014 Nov 13;371(20):1889-99

Efectos de la vacunación en Sudáfrica sobre la enfermedad invasiva neumocócica

Effects of vaccination on invasive pneumococcal disease in South Africa

von Gottberg $A$, et al.

Comentario: Dra. Ángela Gentile. Hospital de Niños Ricardo Gutiérrez, Buenos Aires.

JAMA 2014;312(7):712-718. doi:10.1001/JAMA.2014.8637

Efecto de la oximetría en la internación de niños con bronquiolitis. Un estudio clínico randomizado

Effect of oximetry on hospitalization in bronchiolitis. A randomized clinical trial

Schuh $S$, et al.

Comentario: Dra. Hilda Giugno. Neumonología, Hospital de Pediatría Prof. Dr. Juan P. Garrahan. 